\title{
Measurement of oral health quality of life among patients who underwent haematopoietic stem-cell transplantation
}

\section{Nayara Fernanda PEREIRA(a) Paula Verona Ragusa da SILVA(b) Cíntia Yuki FUKUOKA(c) Edgard MICHEL-CROSATO(a) Alyne Simões GONÇALVES(c) Fábio Abreu ALVES(b) Garles Miller Matias VIEIRA ${ }^{(d)}$ Maria Gabriela Haye BIAZEVIC(a)}

(a) Universidade de São Paulo - USP, School of Dentistry, Community Dentistry Department, São Paulo, SP, Brazil.

(b) Universidade de São Paulo - USP, School of Dentistry, Stomatology Department, São Paulo, SP, Brazil.

(c) Universidade de São Paulo - USP, School of Dentistry, Biomaterials and Oral Biochemistry Department, São Paulo, SP, Brazil.

(d) AC Camargo Cancer Center, Clinical Oncology Department, São Paulo, SP, Brazil.

Declaration of Interests: The authors certify that they have no commercial or associative interest that represents a conflict of interest in connection with the manuscript.

Corresponding Author: Maria Gabriela Haye Biazevic E-mail:biazevic@usp.br

Submitted: August 18, 2017

Accepted for publication: May 21, 2018

Last revision: June 08, 2018
Abstract: Oral mucositis is a painful condition that occurs in patients who have undergone haematopoietic stem-cell transplantation (HSCT) and has a huge impact on their quality of life. The objective of this study was to examine the extent to which interleukin-6 (IL-6) and tumour necrosis factor alpha (TNF-a) are associated with oral health quality of life among patients who underwent HSCT. A. C. Camargo Cancer Center patients were evaluated over 20 months at 4 different timepoints: a) at patient admission (M1); b) on the day of infusion of HSC (M2); c) 12 and 20 days after the first day of the conditioning regimen for autologous and allogeneic transplantation, respectively (M3); and d) 30 days after the first day of the conditioning regimen (M4). Mucositis clinical evaluations were performed using World Health Organization (WHO) criteria. Oral health quality of life was measured using the Oral Health Impact Profile short form (OHIP-14), Oral Mucositis Quality of Life (OMQoL) and Patient-Reported Oral Mucositis Symptom (PROMS) scales. Correlations between clinical data and quality of life scores were examined. STATA 11.0 was used to perform the statistical analyses (5\% level of significance). Eighty-two patients participated in the study; $62.2 \%$ were male, $28.05 \%$ had multiple myeloma, and the mean age was 48.49 years (SD 13.76). Higher scores (worse quality of life) were observed as mucositis scores increased, but the results were not significant. The OMQoL had a high correlation with OHIP-14 (0.8377), but the correlation between the PROMS scale and the OHIP-14 was lower (0.6643). Higher concentrations of IL-6 and TNF-a were associated with worse quality of life, according to all indices $(p<0.05)$. Oral mucositis was associated with quality of life scores $(p<0.01)$.

Keywords: Stomatitis; Quality of Life; Saliva.

\section{Introduction}

Most patients who have undergone haematopoietic stem-cell transplantation (HSCT) are diagnosed with lymphomas and leukaemia. In 2012, 566,000 new cases of lymphomas with 305,000 deaths and 352,000 new cases of leukaemia with 265,000 deaths occurred worldwide. ${ }^{1}$

Oral mucositis is a painful condition that can affect patients who have undergone HSCT and has an impact on their quality of life. ${ }^{2}$ The pathogenesis of the condition presents several challenges that can interfere 
with patient prognosis. Pro-inflammatory cytokines, especially tumour necrosis factor alpha (TNF-a) and interleukin-6 (IL-6), have been associated with fever and chronic and acute inflammatory reactions. ${ }^{3}$ IL-6 is a cytokine that acts on many systems of the body. High blood levels have been associated with worse prognosis and survival rates among patients diagnosed with non-Hodgkin's lymphoma. ${ }^{4}$ Quality of life might be affected by these conditions, since worse clinical conditions will impact the patient's quality of life, such as through their ability to eat, to swallow or to interact to other persons socially.

In this context, we did not find a study that had considered both oral health quality of life and salivary levels of IL- 6 and TNF- $\alpha$. Therefore, the objective of this investigation was to verify the possible associations between IL- 6 and TNF- $\alpha$ levels and oral health quality of life among patients who have undergone HSCT.

\section{Methodology}

Adult patients (18 years old and older) who underwent HSCT at the A. C. Camargo Cancer Center from August 2013 to May 2015 were invited to participate. HSCT patients who were 18 years of age or older were included in the study, and patients who refused to participate after being invited and those with no medical conditions indicated on the questionnaires were excluded from the study.

As per the hospital routine, patients underwent preventive low-energy laser therapy in their oral mucosa with a gallium aluminium arsenate (GaAlAs) diode laser (Twin laser, MM Optics ${ }^{\circledR}$, MM Optics Ltda., São Carlos, São Paulo, Brazil) at a continuous wavelength of $660 \mathrm{~nm}$, with $15 \mathrm{~mW}$ power and $3.8 \mathrm{~J} / \mathrm{cm}^{2}$ energy density. The laser illuminated points with $1.0 \mathrm{~cm}$ distance between them, bilaterally covering the buccal mucosa, the lower and upper labial vestibule, the lower and upper labial mucosa, the ventral and lateral tongue, and the floor of the mouth. Each point was illuminated for $10 \mathrm{~s}$. Irradiation was initiated at the beginning of the conditioning regimen and was completed on the second day after stem cell transplantation $($ day +2$)$. After the prophylactic laser protocol, patients were followed daily. When mucositis grade $\geq 2$ was detected, a therapeutic laser therapy was applied using the same protocol as that used in prophylactic therapy but irradiating only the area containing lesions. This treatment was repeated daily until pain remission. To avoid retinal damage, the patients wore $660-\mathrm{nm}$ wavelength-specific glasses.

The Oral Health Impact Profile short form (OHIP-14) was used to evaluate general oral health-related quality of life. ${ }^{5}$ The Brazilian version of the instrument was used. ${ }^{6}$ This instrument contains 14 questions related to five dimensions: functional limitations, physical pain, psychological limitations, social limitations and difficulties in performing daily activities.

To evaluate oral mucositis and how it affected the patients' quality of life, two instruments were used: the Oral Mucositis Quality of Life (OMQoL) assessment ${ }^{7}$ and the Patient-Reported Oral Mucositis Symptom (PROMS) scale, ${ }^{8}$ both of which were freely translated to the Brazilian Portuguese language.

The OMQoL assessment is composed of 31 questions that highlight the symptoms of oral mucositis using a Likert scale. The PROMS scale consists of 10 questions that patients answer by indicating their responses regarding the intensity of some symptoms on a 10-centimetre-long straight line.

Oral mucositis was clinically evaluated using the World Health Organization (WHO) criteria ${ }^{9}$ as follows: grade 0 indicated no symptoms; grade 1 indicated soreness/erythema, but no ulcers in the oral cavity; grade 2 indicated erythema and ulcers, but able to eat solids; grade 3 indicated ulcers requiring a liquid diet; and grade 4 indicated that oral alimentation was not possible.

Salivary analyses were performed for all patients. ${ }^{10,11}$ Patients were asked not to ingest food or beverages and not to clean their mouths for 60 minutes prior to saliva collection. After rinsing the mouth with distilled water, parafilm-stimulated saliva was collected for 5 minutes. The samples were classified and stored at $-80^{\circ} \mathrm{C}$ until the biochemical analysis was performed.

TNF- $\alpha$ and IL-6 were quantified using a Human TNF-alpha Quantikine ELISA kit ${ }^{\circledR}$ and a Human IL-6 Quantikine ELISA kit ${ }^{\circledR}$, respectively (R\&D Systems ${ }^{\circledR}$, Minneapolis, Minnesota, USA). In these ELISA kits, the standards and samples are added to a microplate containing the monoclonal antibody specific for TNF- $\alpha$ or IL-6, and any TNF- $\alpha$ or IL- 6 present in the 
solution is bound by the antibody. After washing, an enzyme-linked polyclonal antibody is added to the wells, they are washed again, and a substrate solution is added. After stopping the reaction, the intensity of the colour, which is proportional to the amount of TNF-a or IL- 6 bound in the initial step, is measured. The IL- 6 and TNF- a quantification was dichotomized after the median value was calculated.

All of the measures used (quality of life evaluation using 3 instruments, clinical mucositis grading and saliva collection) were performed at four timepoints for each patient: Moment 1 (M1) was at patient admission; Moment 2 (M2) was on the day of HSCT; Moment 3 (M3) was 12 and 20 days after the first day of the conditioning regimen following autologous and allogeneic transplantation, respectively; and Moment 4 (M4) was at patient discharge.

Data were organized using an Excel $^{\circledR}$ (Microsoft, Redmond, Washington, USA) spreadsheet and were analysed using STATA $13.0^{\circledR}$ (StataCorp LP, College Station, Texas, USA). The Kolmogorov-Smirnoff test was used to verify a normal distribution. ANOVAs were used for repeated measures data with Bonferroni adjustments. Spearman correlations were used to verify the degree of agreement among the quality of life instruments, and t-tests were used to examine the mean differences between salivary cytokines and quality of life. All tests were evaluated at a 5\% level of significance.

This investigation was performed in accordance with the international guidelines for investigations involving human beings and the Helsinki Declaration. The project was approved by the ethics committees of the A. C. Camargo Cancer Center and the University of São Paulo's School of Dentistry (Process number 439.910). All participants were informed of the investigation objectives and procedures. After being invited to participate, the patients signed informed consent forms.

\section{Results}

Eighty-two (82) patients participated in the study; $62.2 \%$ of them were male, and $28.05 \%$ had multiple myeloma (Table 1). The mean age was 48.49 years (SD 13.76) and ranged from 18 to 67 years. Most of the patients were submitted to autologous transplant (60.98\%) (Table 1).

Table 2 describes mucositis grades and quality of life scores using the OHIP-14, OMQoL and PROMS assessments. Higher scores (worse quality of life) were observed as mucositis scores increased (Table 2 and Figure). Furthermore, most of the quality of life results differed significantly from the others (Table 2).

Table 3 outlines the correlations among quality of life instruments. The OMQoL assessment was highly correlated with the OHIP-14 (0.8377), but the correlation between the PROMS scale and the OHIP-14 was lower (0.6643).

Table 4 shows the results of IL- 6 and TNF-a levels at the moments of treatment. Higher concentrations of IL-6 and TNF-a were not associated with worse quality of life using any of the instruments used (Table 5). Differences between the groups were only observed in the PROMS scale and IL-6 concentrations $(\mathrm{p} \leq 0.020)$ (Table 5).

\section{Discussion}

More patients who underwent autologous rather than allogeneic transplant participated in the study. Haematologic diseases usually affect a wide range of ages, from infants to the elderly. ${ }^{1}$ In our study, the age range was 18 to 67 years, and

Table 1. Descriptive analysis of the participants.

\begin{tabular}{lcc}
\hline Varible & $\mathrm{n}$ & $\%$ \\
\hline Gender & & \\
Male & 51 & 62.20 \\
Female & 31 & 37.80 \\
Diagnostic & & \\
Lymphoma & 31 & 37.80 \\
Multiple myeloma & 23 & 28.05 \\
Leukaemia & 21 & 25.61 \\
Other & 7 & 8.54 \\
Transplant type & & \\
Autologous & 50 & 60.98 \\
Allogeneic & 32 & 39.02 \\
Age & Mean (SD) & Min/Max \\
in years & $48.49(13.76)$ & $18-67$ \\
\hline
\end{tabular}


Table 2. Mucositis and quality of life based on different measurement instruments.

\begin{tabular}{|c|c|c|c|c|}
\hline Mucositis & \multirow{2}{*}{$n$} & \multirow{2}{*}{ OHIP-14* } & \multirow{2}{*}{ SD } & Different $(p<0.05)^{\#}$ \\
\hline (WHO) & & & & from factor $n r^{\# \#}$ \\
\hline (1) Grade 0 & 196 & 0.36 & 0.62 & $(2)(3)(4)(5)$ \\
\hline (2) Grade 1 & 65 & 0.88 & 0.90 & $(1)(3)(4)(5)$ \\
\hline (3) Grade 2 & 13 & 1.48 & 0.87 & $(1)(2)(4)$ \\
\hline (4) Grade 3 & 12 & 2.65 & 0.85 & $(1)(2)(3)$ \\
\hline (5) Grade 4 & 1 & 2.79 & $?$ & $(1)(2)$ \\
\hline Mucositis & \multirow{2}{*}{$\mathrm{n}$} & \multirow{2}{*}{ OMQoL** } & \multirow{2}{*}{ SD } & Different $(p<0.05)^{*}$ \\
\hline (WHO) & & & & from factor $n r^{* *}$ \\
\hline (1) Grade 0 & 196 & 40.57 & 13.88 & $(2)(3)(4)(5)$ \\
\hline (2) Grade 1 & 66 & 52.42 & 17.97 & $(1)(3)(4)(5)$ \\
\hline (3) Grade 2 & 13 & 63.15 & 23.89 & $(1)(2)(4)(5)$ \\
\hline (4) Grade 3 & 12 & 94.58 & 17.67 & $(1)(2)(3)$ \\
\hline (5) Grade 4 & 1 & 113.00 & $?$ & $(1)(2)(3)$ \\
\hline Mucositis & \multirow{2}{*}{$n$} & \multirow{2}{*}{ PROMS*** } & \multirow{2}{*}{ SD } & Different $(p<0.05)^{*}$ \\
\hline (WHO) & & & & from factor $\mathrm{nr}^{* *}$ \\
\hline (1) Grade 0 & 190 & 0.45 & 0.75 & $(2)(3)(4)(5)$ \\
\hline (2) Grade 1 & 66 & 1.90 & 2.16 & $(1)(3)(4)(5)$ \\
\hline (3) Grade 2 & 13 & 4.31 & 2.69 & $(1)(2)(4)$ \\
\hline (4) Grade 3 & 12 & 6.88 & 1.57 & $(1)(2)(3)$ \\
\hline (5) Grade 4 & 1 & 6.32 & $?$ & $(1)(2)$ \\
\hline
\end{tabular}

valid questionnaires $=287 ;{ }^{* *}$ valid questionnaires $=288 ;{ }^{* * *}$ valid questionnaires $=282 .{ }^{*}$ ANOVA.; ${ }^{\# \#}$ numbers in the same line mean differences among the groups.

one of the inclusion criteria was being 18 years old or older. Not all patients were available to respond to the questionnaires at all study timepoints for clinical reasons. Therefore, the total number of valid responses is indicated in Table 2.

Mucositis degree was associated with worse quality of life. This result was expected, as higher mucositis grades affect feeding ability. This finding was also observed in another published study. ${ }^{2,12}$ At the $3^{\text {rd }}$ treatment timepoint, as expected, worse quality of life and degree of mucositis were found. Cytokine levels were not different across the timepoints (as observed on Table 4). For that reason, and because of the limited amount of valid measurement data, the results were presented together. This issue is a potential limitation of this study.

All three instruments produced good results. The OHIP-14 is generic, as it was created to highlight several oral conditions that have the potential to affect a patient's quality of life. The other 2 instruments (OMQoL and PROMS scale) more specifically targeted oral mucositis symptoms among patients receiving several cancer treatments.

The advantage of using a more generic instrument to measure quality of life (i.e., OHIP-14) is that the impact of some morbidities experienced by the patients can be compared to the impact on other patients suffering from other diseases. In this way, it is possible to analyse which oral health conditions have the potential to affect more patients than others. ${ }^{5}$ A disadvantage of this instrument is that the patient's score can be attributable to other oral health conditions that might not have resulted specifically from the treatment (i.e., HSCT). However, this limitation can be minimized depending on the hospital's protocol with regard to oral health. It is known that having a dental team included as part of the medical team can minimize the effects of previous oral health 

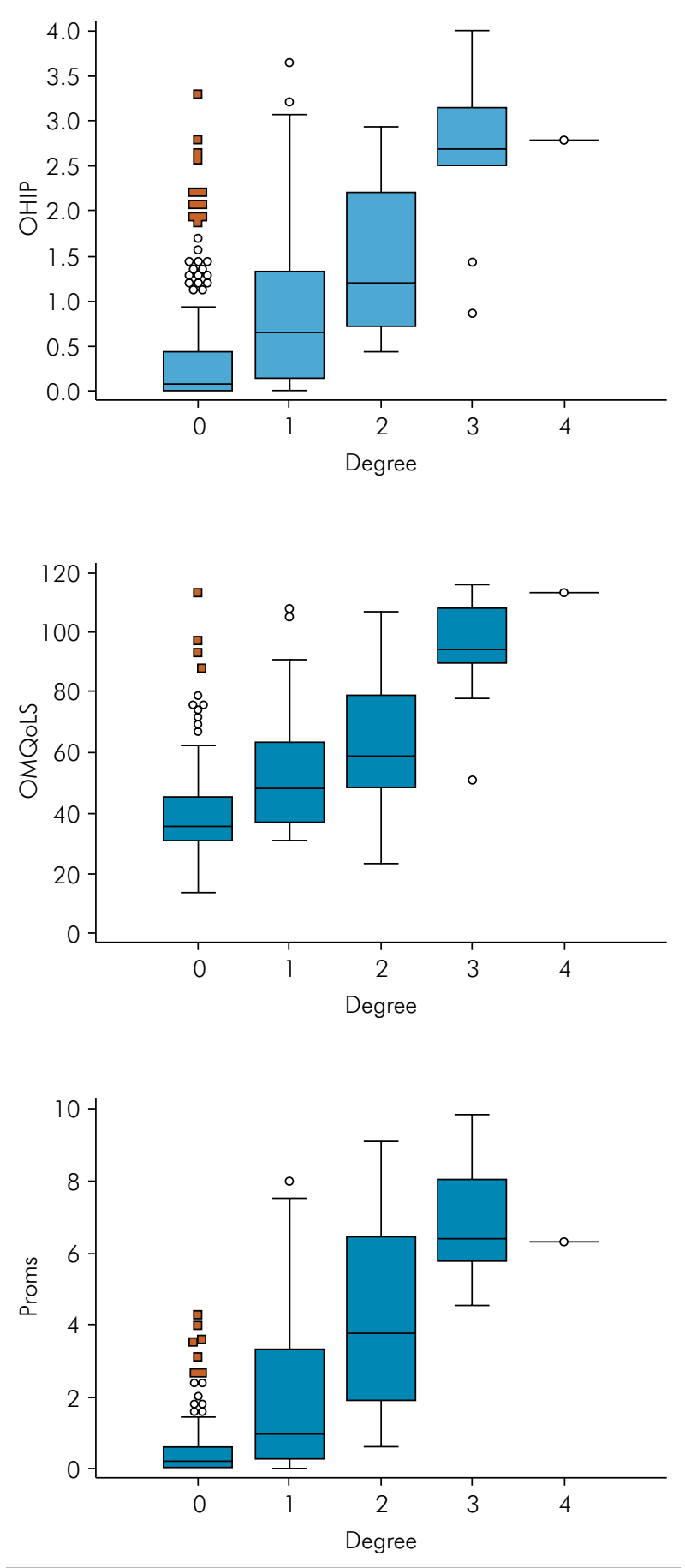

Figure 1. Mucositis and quality of life instrument correlations. 1A-Mucositis and OHIP-14. 1B-Mucositis and OMQoL. 1CMucositis and PROMS scale.

conditions on HSCT. Our investigation had this support, as the hospital used a protocol both before and during conditioning in which all patients had their oral health needs treated.
Table 3. Correlations among the OHIP-14, OMQoL and PROMS instruments*.

\begin{tabular}{lccc}
\hline & OHIP-14 & OMQOL & PROMS \\
\hline OHIP-14 & 10.000 & - & - \\
OMQOL & 0.8377 & 10.000 & - \\
PROMS & 0.6643 & 0.7346 & 10.000 \\
\hline *ANOVA & & &
\end{tabular}

The other 2 instruments (the OMQoL and the PROMS scale) were created specifically to detect alterations in oral health quality of life among patients with cancer; oral mucositis is usually one of the most painful conditions. ${ }^{78}$ Therefore, their results (scores) are due to their symptoms related to this systemic condition (in our investigation, in response to the HSCT conditioning). As a disadvantage, it is possible that comparisons with other diseases and oral conditions are not appropriate, as most of the questions focus on oral mucositis symptoms. In fact, another study found that, for several cancer therapies, the OMQoL instrument better correlated than another instrument (the European Organization for Research and Treatment of Cancer Quality of Life questionnaire, EORTC QLQ-C30, Chinese version) designed to address mucositis symptoms experienced by the patients. ${ }^{13}$ The OMQoL is being validated for the Brazilian Portuguese language, and the PROMS scale was freely translated to perform this investigation, which is a limitation of this study.

When planning to perform cytokine quantification, we expected to observe that higher concentrations of IL-6 and TNF-a were correlated with higher quality of life scores. However, our results did not reveal substantial differences between the groups with higher cytokine concentrations and those with lower ones. Only the PROMS scale and IL-6 were related. Future studies should be designed to verify if the patients' experiences with their oral condition predict higher cytokine concentrations in saliva. ${ }^{14,15}$

We should mention that these results come from a specific sample of patients who underwent HSCT in one hospital in São Paulo, Brazil. Even though the city has 12 million inhabitants, and patients from several parts of the country attend to be treated, generalization 
Measurement of oral health quality of life among patients who underwent haematopoietic stem-cell transplantation

Table 4. Interleukin-6 (IL-6) (pg/mg protein) and tumour necrosis factor alpha (TNF-a) (pg/mg protein) levels at the 4 treatment timepoints*.

\begin{tabular}{|c|c|c|c|c|c|c|c|c|c|c|c|c|c|c|c|c|c|}
\hline \multirow{2}{*}{ Variable } & \multicolumn{4}{|c|}{ MI } & \multicolumn{4}{|c|}{ M2 } & \multicolumn{4}{|c|}{ M3 } & \multicolumn{5}{|c|}{ M4 } \\
\hline & $\mathrm{n}$ & mean & median & SD & $\mathrm{n}$ & mean & median & SD & $\mathrm{n}$ & mean & median & SD & $\mathrm{n}$ & mean & median & SD & $p$ \\
\hline IL-6 & 67 & 0.99 & 0.43 & 3.00 & 49 & 1.94 & 0.57 & 4.82 & 30 & 1.12 & 0.99 & 0.82 & 34 & 0.62 & 0.46 & 0.48 & 0.245 \\
\hline TNF- $a$ & 54 & 0.24 & 0.11 & 0.34 & 41 & 0.20 & 0.09 & 0.31 & 35 & 0.40 & 0.18 & 0.45 & 27 & 0.25 & 0.14 & 0.29 & 0.078 \\
\hline
\end{tabular}

* ANOVA.

Table 5. Correlations between interleukin-6 (IL-6) (pg/mg protein) and tumour necrosis factor alpha (TNF-a) (pg/mg protein) and quality of life indicators.

\begin{tabular}{|c|c|c|c|c|c|c|c|}
\hline \multirow{2}{*}{ Variable } & \multicolumn{3}{|c|}{ IL-6 (0.00 to 0.5) } & \multicolumn{3}{|c|}{ IL-6 $(0.6+)$} & \multirow{2}{*}{$p \leq * *$} \\
\hline & $\mathrm{n}^{*}$ & Mean & SD & $\mathrm{n}^{*}$ & Mean & SD & \\
\hline OHIP-14 & 86 & 7.06 & 10.72 & 94 & 8.36 & 11.94 & 0.44 \\
\hline OMQoL & 86 & 43.38 & 16.63 & 94 & 47.13 & 18.40 & 0.15 \\
\hline PROMS & 86 & 0.56 & 1.10 & 91 & 1.16 & 2.07 & 0.02 \\
\hline \multirow{2}{*}{ Variable } & \multicolumn{3}{|c|}{ TNF-a (0.00 a 0.2) } & \multicolumn{3}{|c|}{ TNF-a $(0.2+)$} & \\
\hline & $n$ & Mean & SD & N & Mean & SD & $\mathrm{p} \leq^{*}$ \\
\hline OHIP-14 & 99 & 8.10 & 11.41 & 42 & 11.69 & 14.76 & 0.12 \\
\hline OMQoL & 99 & 45.14 & 17.33 & 43 & 51.14 & 23.88 & 0.09 \\
\hline PROMS & 97 & 0.90 & 1.73 & 43 & 1.39 & 2.32 & 0.17 \\
\hline
\end{tabular}

* number of valid measures; ${ }^{* *}$ ANOVA

of the results must be cautious. Furthermore, since all patients received low-energy laser therapy as a preventive measure, the results may have been influenced by this protocol. More studies are necessary to verify which aspects of the patients experience with HSCT affect their quality of life and in which ways medical teams can use these results to treat patients in a holistic manner.

\section{References}

1. Stewart BW, Wild CP, editors. World cancer report 2014. Lyon: IARC; 2014.

2. Bezinelli LM, Eduardo FP, Neves VD, Correa L, Lopes RM, Michel-Crosato E et al. Quality of life related to oral mucositis of patients undergoing haematopoietic stem cell transplantation and receiving specialised oral care with low-level laser therapy: a prospective observational study. Eur J Cancer Care (Engl). 2016 Jul;25(4):668-74. https://doi.org/10.1111/ecc.12344

3. Sonis ST. Mucositis: the impact, biology and therapeutic opportunities of oral mucositis.

\section{Acknowledgments}

The investigators would like to acknowledge the State of São Paulo's Research Fund (FAPESP, process number 2012/22434-0) and the National Council for Investigation (CNPq, process number 146336/2014-3), which provided the funding to perform this investigation.
Oral Oncol. 2009 Dec;45(12):1015-20.

https://doi.org/10.1016/i.oraloncology.2009.08.006

4. Tegg EM, Griffiths AE, Lowenthal RM, Tuck DM, Harrup R, Marsden KA et al. Association between high interleukin- 6 levels and adverse outcome after autologous haemopoietic stem cell transplantation. Bone Marrow Transplant. 2001 Nov;28(10):929-33. https://doi.org/10.1038/sj.bmt.1703272

5. Slade GD. Derivation and validation of a short-form oral health impact profile. Community Dent Oral Epidemiol. 1997 Aug;25(4):284-90. https://doi.org/10.1111/j.1600-0528.1997.tb00941.x 
6. Oliveira BH, Nadanovsky P. Psychometric properties of the Brazilian version of the Oral Health Impact Profile-short form. Community Dent Oral Epidemiol. 2005 Aug;33(4):307-14. https://doi.org/10.1111/j.1600-0528.2005.00225.x

7. Cheng KK, Leung SF, Thompson DR, Tai JW, Liang RH, Kan AS et al. New measure of health-related quality of life for patients with oropharyngeal mucositis: development and preliminary psychometric evaluation. Cancer. 2007 Jun;109(12):2590-9. https://doi.org/10.1002/cncr.22730

8. Kushner JA, Lawrence HP, Shoval I, Kiss TL, Devins GM, Lee L et al. Development and validation of a Patient-Reported Oral Mucositis Symptom (PROMS) scale. J Can Dent Assoc. 2008 Feb;74(1):59.

9. World Health Organization. Handbook for reporting results of cancer treatment. Geneva: World Health Organization; 1979.

10. Rüping MJ, Keulertz C, Vehreschild JJ, Lövenich H, Söhngen $D$, Wieland $U$ et al. Association of HSV reactivation and pro-inflammatory cytokine levels with the severity of stomatitis after BEAM chemotherapy and autologous SCT. Support Care Cancer. 2011 Aug;19(8):1211-6. https://doi.org/10.1007/s00520-010-0940-8

11. Navazesh M, Kumar SK. Measuring salivary flow: challenges and opportunities. J Am
Dent Assoc. 2008 May;139 Suppl:35S-40S. https://doi.org/10.14219/jada.archive.2008.0353

12. Patussi C, Sassi LM, Munhoz EC, Zanicotti RT, Schussel JL. Clinical assessment of oral mucositis and candidiasis compare to chemotherapic nadir in transplanted patients. Braz Oral Res. 2014;28(1):1-7. https://doi.org/10.1590/1807-3107BOR-2014.vol28.0050

13. Cheng KK, Leung SF, Liang RH, Tai JW, Yeung RM, Thompson DR. A patient-reported outcome instrument to assess the impact of oropharyngeal mucositis on health-related quality of life: a longitudinal psychometric evaluation. Support Care Cancer. 2009 Apr;17(4):389-98. https://doi.org/10.1007/s00520-008-0485-2

14. Morales-Rojas T, Viera N, Morón-Medina A, Alvarez CJ, Alvarez A. Proinflammatory cytokines during the initial phase of oral mucositis in patients with acute lymphoblastic leukaemia. Int J Paediatr Dent. 2012 May;22(3):191-6. https://doi.org/10.1111/j.1365-263X.2011.01175.x

15. Silva GB, Sacono NT, Othon-Leite AF, Mendonça EF, Arantes $A M$, Bariani $C$ et al. Effect of low-level laser therapy on inflammatory mediator release during chemotherapy-induced oral mucositis: a randomized preliminary study. Lasers Med Sci. 2015 Jan;30(1):117-26. https://doi.org/10.1007/s10103-014-1624-2 